\title{
Pattern-recognition-based Identification of Proteases and Their Complexes by a One-component Array Composed of a Dansyl-modified Charged Polymer
}

\author{
Shunsuke Tomita ${ }^{1,2^{*}}$ and Ryoji Kurita ${ }^{1,2,3 * *}$ \\ ${ }^{1}$ Health and Medical Research Institute, National Institute of Advanced Industrial Science and Technology, \\ 1-1-1 Higashi, Tsukuba, Ibaraki 305-8566, Japan \\ ${ }^{2}$ DBT-AIST International Laboratory for Advanced Biomedicine (DAILAB), DBT-AIST International Center for \\ Translational \& Environmental Research (DAICENTER), National Institute of Advanced Industrial Science and \\ Technology, 1-1-1 Higashi, Tsukuba, Ibaraki 305-8566, Japan \\ ${ }^{3}$ Faculty of Pure and Applied Sciences, University of Tsukuba, 1-1-1 Tennodai, Tsukuba, Ibaraki 305-8573, Japan
}

(Received October 22, 2020; accepted December 14, 2020)

Keywords: proteases, biosensors, multivariate analysis, polymers

Pattern-recognition-based protein sensing has recently attracted attention as an alternative to conventional specificity-based sensing. Herein, we present a simple one-component array consisting of a charged poly-L-lysine modified with environment-responsive dansyl fluorophores (PLL-Dnc) for the identification of proteases. An array of PLL-Dnc dissolved in three buffer solutions with different $\mathrm{pH}$ values is capable of producing fluorescent patterns that are unique to each protease via diverse interactions, allowing the identification of eight proteases by multivariate analysis. Although it is impractical to quantify mixed samples of proteases and their inhibitor proteins by conventional enzyme-activity-based assays, the present array has enabled the discrimination of mixtures of trypsin and $\alpha_{1}$-antitrypsin (ATT) with different compositions. Considering that the PLL-Dnc array has high accessibility and consists of only a single polymeric material that can be easily prepared, our method is expected to allow screening for the formation of protein-protein complexes, as well as the detection of diseaseassociated proteases.

\section{Introduction}

Proteases have the ability to regulate the physiological functions of proteins through enzymatic hydrolysis, and are therefore closely involved in biological processes. Abnormal protease activity causes a variety of diseases, such as cancer and inflammatory diseases; therefore, the detection of proteases is of critical importance for the diagnosis and treatment of medical conditions. ${ }^{(1,2)}$ For the detection of proteases, specificity-based methods are generally used, such as enzyme-linked immunosorbent assays (ELISAs) using antibodies and enzymecatalyzed reactions. ${ }^{(1,3,4)}$ However, it is difficult to develop antibodies or enzyme substrates for highly specific detection, and the production of these molecules is costly and laborious.

\footnotetext{
*Corresponding author: e-mail: s.tomita@aist.go.jp

${ }^{* *}$ Corresponding author: e-mail: r.kurita@aist.go.jp

https://doi.org/10.18494/SAM.2021.3074
} 
Pattern-recognition-based sensing techniques have attracted considerable attention as an alternative approach to the easy and accurate identification of various biological analytes, including proteins and cells. ${ }^{(5)}$ This sensing strategy utilizes an array of multiple molecules that are nonspecific but have different affinities, i.e., cross-reactivity, with the analytes. In a typical form, the molecules are modified with reporter units, such as chromophores and fluorophores, which change their optical properties upon binding to the analytes. Response readout after mixing such an array with the analytes produces unique optical patterns of the individual analytes. The resulting patterns are then processed by multivariate analysis techniques to accurately identify the analytes. To date, much effort has been devoted to the development of arrays for protein sensing using various cross-reactive materials, including supramolecular complexes $^{(6-8)}$ and nanoparticles. ${ }^{(9,10)}$ Recently, we have developed pattern-recognition-based sensing systems for protease detection using arrays of fluorophore-modified DNAs with different sequences. ${ }^{(11,12)}$

To bring the pattern-recognition-based protease sensors closer to practical use, it is desirable for the sensing system to be easily constructed with fewer materials. Here, we report an array consisting of only a single material for protease detection. The system is based on our recent studies $^{(13,14)}$ on protein and cell sensing using a poly-L-lysine modified with environmentresponsive dansyl fluorophores (PLL-Dnc; Fig. 1). PLL is a suitable scaffold for the detection of macromolecules such as proteases because it contains positively charged amino groups $\left(\mathrm{p} K_{\mathrm{a}} \sim 10.5\right)$ and hydrophobic $n$-butyl groups, which provide the ability to bind strongly and nonspecifically to proteases through multiple electrostatic and hydrophobic interactions. When PLL-Dnc binds to a protease, the dansyl fluorescence changes and, in many cases, increases depending on the nature of the binding sites in the protease. As the dominant forces between PLL-Dnc and proteases differ with the $\mathrm{pH}$ value of the solution, an array of PLL-Dnc dissolved in different buffer solutions produces fluorescence patterns specific to the individual proteases
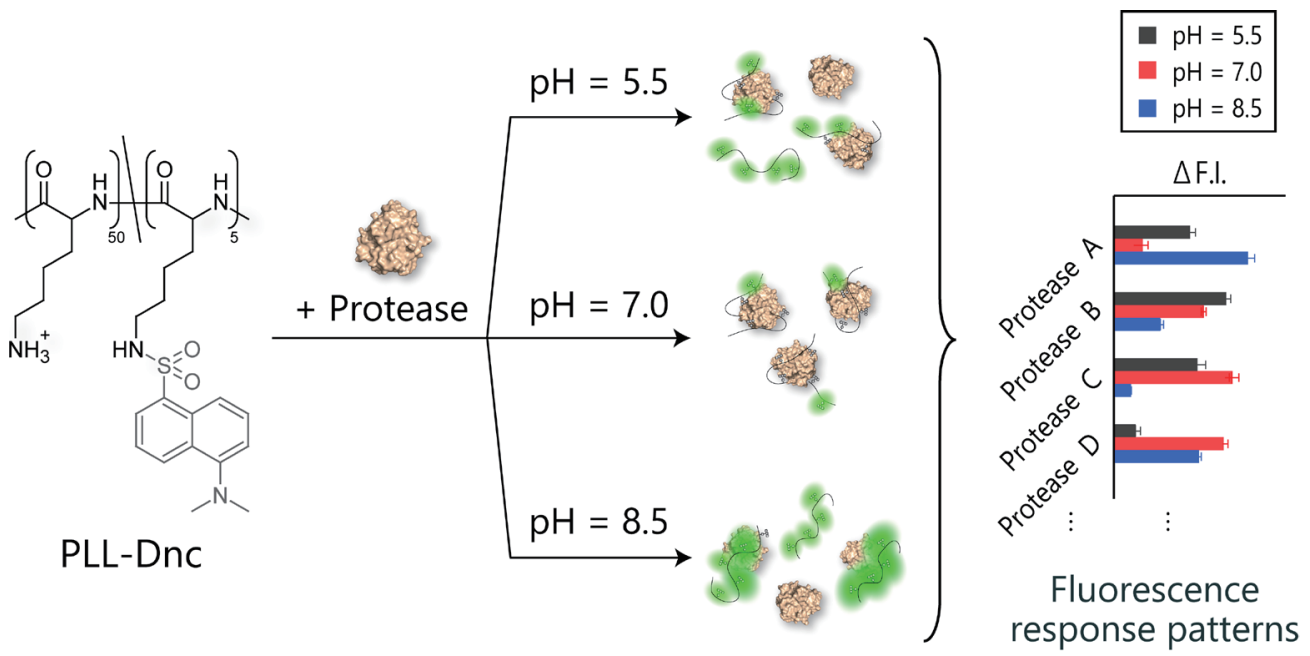

Fig. 1. (Color online) Schematic representation of the generation of fluorescence response patterns of proteases using an array of an environment-responsive PLL-Dnc. 
(Fig. 1). Multivariate analysis of the patterns obtained from a simple array consisting of a single PLL-Dnc successfully identified eight different proteases, as well as the compositions of mixtures of a protease and its inhibitor.

\section{Materials and Methods}

\subsection{Materials}

Carboxypeptidase A from bovine pancreas (Car), $\alpha$-chymotrypsin from bovine pancreas (Chy), elastase from porcine pancreas (Ela), papain from papaya latex (Pap), proteinase $\mathrm{K}$ from Tritirachium album (Pro), thrombin from bovine plasma (Thr), trypsin from bovine pancreas (TryB), trypsin from porcine pancreas (TryP), $\alpha_{1}$-antitrypsin from human plasma (ATT), 3-morpholinopropanesulfonic acid (MOPS), and 2-morpholinoethanesulfonic acid (MES) were purchased from Sigma-Aldrich, Co., LLC (St. Louis, MO, USA). 3-[4-(2-Hydroxyethyl)-1piperazinyl] propanesulfonic acid (EPPS) was purchased from Dojindo Molecular Technologies, Inc. (Kumamoto, Japan). Poly-L-lysine trifluoroacetate (PLL; degree of polymerization: 55) was purchased from Alamanda Polymers, Inc. (Huntsville, AL, USA). The PLL-Dnc polymer (5 out of 55 lysine units per side chain were modified with Dnc groups) has already been reported. ${ }^{(14)}$

\subsection{Fluorescence measurements}

The concentration of proteases and ATT was determined from their absorbance on a NanoDrop 1000 spectrophotometer (Thermo Scientific, Inc., Waltham, MA, USA) using the extinction coefficients given in a previous report. ${ }^{(11)}$ Fluorescence measurements were performed on a Cytation5 Imaging Reader (BioTek Instruments, Inc., Winooski, VT, USA). Solutions $(120 \mu \mathrm{L})$ containing $5.0 \mu \mathrm{g} / \mathrm{mL}$ PLL-Dnc and $0-20 \mu \mathrm{g} / \mathrm{mL} \mathrm{Thr}$ in $20 \mathrm{mM}$ MES buffer $(\mathrm{pH}=5.0)$ were prepared in each well of a 96-well black microplate (Corning Inc., Corning, NY, USA) using a PIPETMAX liquid handling system (Gilson Inc., Middleton, WI, USA). After incubation $\left(35^{\circ} \mathrm{C}, 10 \mathrm{~min}\right)$, the fluorescence intensity was recorded at $35{ }^{\circ} \mathrm{C}$ at $\lambda_{e x} / \lambda_{e m}=340 \mathrm{~nm} / 520 \mathrm{~nm}$.

\subsection{Pattern-recognition-based sensing}

Aliquots $(108 \mu \mathrm{L})$ of PLL-Dnc $(5.5 \mu \mathrm{g} / \mathrm{mL})$ in $22.2 \mathrm{mM}$ MES buffer $(\mathrm{pH}=5.5), 22.2 \mathrm{mM}$ MOPS buffer ( $\mathrm{pH}=7.0)$, or $22.2 \mathrm{mM}$ EPPS buffer $(\mathrm{pH}=8.5)$ were deposited in the wells of a 96-well plate using a PIPETMAX system. After incubation at $35^{\circ} \mathrm{C}$ for $10 \mathrm{~min}$, the fluorescence intensity was recorded in two different channels $\left[\lambda_{e x} / \lambda_{e m}: 320 \mathrm{~nm} / 520 \mathrm{~nm}(\mathrm{Ch} 1)\right.$ and $360 \mathrm{~nm} / 480$ $\mathrm{nm}(\mathrm{Ch} 2)]$. Subsequently, aliquots $(12 \mu \mathrm{L})$ of the analytes in distilled water were added to each well, and the fluorescence intensity was recorded after $10 \mathrm{~min}$ of incubation at $35{ }^{\circ} \mathrm{C}$. This process was repeated six times to generate a data matrix. This data matrix was processed by linear discriminant analysis (LDA) using SYSTAT 13 software (Systat Inc., Evanston, IL, USA). 


\section{Results and Discussion}

\subsection{Pattern-recognition-based protease sensing}

In this study, we employed PLL-Dnc in which 5 out of 55 units of lysine side chains were modified with dansyl groups, on the basis of the results of our previous study. ${ }^{(14)}$ The number of dansyl groups introduced was determined by considering the background fluorescence and the magnitude of the signal of the analytes. ${ }^{(14)}$ Dansyl fluorophores exhibit weak fluorescence in aqueous solutions, which intensifies when the polarity of the surrounding environment decreases upon binding to proteins. ${ }^{(15,16)}$ First, we examined whether PLL-Dnc exhibits fluorescence responses against proteases. Although $\mathrm{Thr}(\mathrm{p} I=7.1$; Table 1) was positively charged at a weakly acidic $\mathrm{pH}$ of 5.5, mixing with cationic PLL-Dnc resulted in an increase in dansyl fluorescence [(Fig. 2(a)]. This result suggests that PLL-Dnc can bind to Thr despite the expected electrostatic repulsion between them, probably owing to the hydrophobic interactions and the heterogeneous amino acid distribution on the Thr surface.

Once we had confirmed that proteases can be detected using PLL-Dnc, we subsequently attempted to discriminate eight different proteases (Table 1). In this sensing procedure, a solution containing each protease was mixed with PLL-Dnc $(5.0 \mu \mathrm{g} / \mathrm{mL})$ to a final protease concentration of $20 \mu \mathrm{g} / \mathrm{mL}$ in three buffer solutions ( $\mathrm{pH}=5.5,7.0$, and 8.5). The net charge of these proteases varied over a selected range of $\mathrm{pH}$ values, which was expected to result in a change in affinity with PLL-Dnc. The fluorescence responses were recorded as $I-I_{0}$, where $I$ and $I_{0}$ are the fluorescence intensities after and before the addition of proteases, respectively, using two detection channels $\left[\lambda_{e x} / \lambda_{e m}=320 \mathrm{~nm} / 520 \mathrm{~nm}(\mathrm{Ch} 1)\right.$ and $\left.360 \mathrm{~nm} / 480 \mathrm{~nm}(\mathrm{Ch} 2)\right]$, given that signal detection at different wavelengths is an easy and effective way to enrich the information available for analyte identification. ${ }^{(17,18)}$ As a result, a dataset of 6 replicates $\times 3 \mathrm{pH}$ values $\times 2$ channels $\times 8$ proteases was generated.

Table 1

Profiles of proteases and the protease inhibitor used in this study.

\begin{tabular}{lcccc}
\hline Analyte & Source & Abbreviation & $\mathrm{p} I$ & $M_{w}\left[\times 10^{3}\right]$ \\
\hline Protease & & & \\
Carboxypeptidase A & Bovine pancreas & Car & 6.0 & 34 \\
Thrombin & Bovine plasma & Thr & 7.1 & 38 \\
Elastase & Porcine pancreas & Ela & 8.5 & 26 \\
$\alpha$-Chymotrypsin & Bovine pancreas & Chy & 8.8 & 25 \\
Proteinase K & Tritirachium album & Pro & 8.9 & 29 \\
Papain & Papaya latex & Pap & 9.6 & 23 \\
Trypsin & Bovine pancreas & TryB & 10.1 & 24 \\
Trypsin & Porcine pancreas & TryP & 10.2 & 23 \\
Protease inhibitor & & & & \\
$\alpha_{1}$-Antitrypsin & Human serum & ATT & 5.4 & 44 \\
\hline
\end{tabular}


(a)

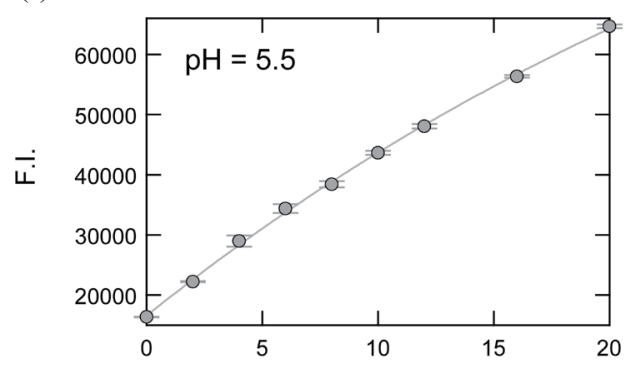

(c)

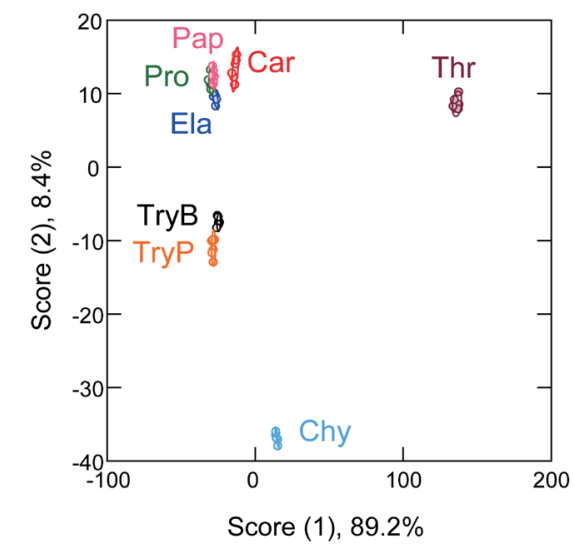

(b)
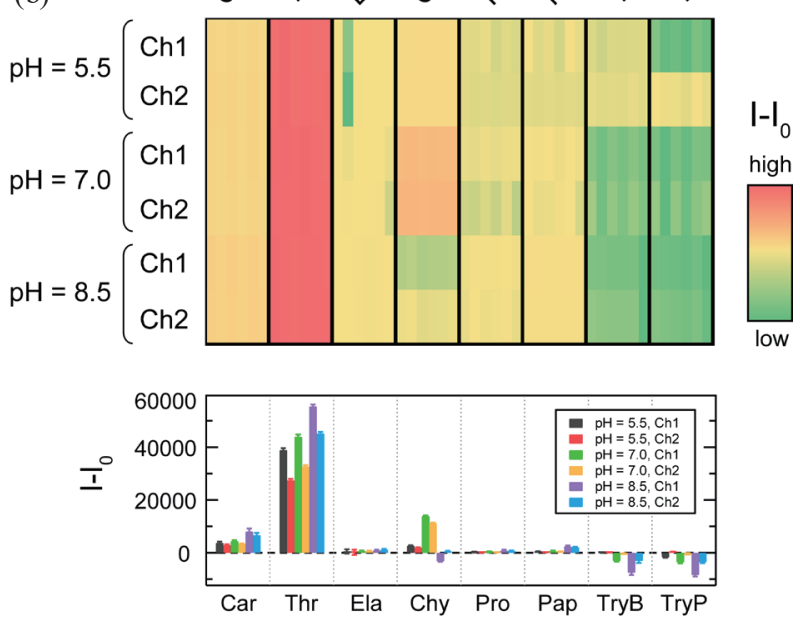

Fig. 2. (Color online) Discrimination of proteases by an array of PLL-Dnc. (a) Binding isotherm for $5.0 \mu \mathrm{g} / \mathrm{mL}$ PLL-Dnc upon addition of Thr in $20 \mathrm{mM}$ MES $(\mathrm{pH}=5.5) . \lambda_{e x}=340 \mathrm{~nm}, \lambda_{e m}=520 \mathrm{~nm}$. Values are shown as mean values $\pm 1 \mathrm{SD}(n=3)$. (b) Heat map and bar graph of the fluorescence response patterns of $20 \mu \mathrm{g} / \mathrm{mL}$ proteases. Six replicates are shown for each analyte. (c) Discriminant score plot for $20 \mu \mathrm{g} / \mathrm{mL}$ proteases. Ellipses represent confidence intervals $( \pm 1 \mathrm{SD})$ for the individual analytes.

The fluorescence responses of the PLL-Dnc array are summarized in the form of a heat map [Fig. 2(b)], which is a color-scaled representation of the fluorescence intensities. As expected, various fluorescence changes were observed depending on the type of protease. The fluorescence patterns were then subjected to LDA to display the complex response patterns as two-dimensional graphics [Fig. 2(c)]. LDA is a supervised multivariate analysis offering insight into data clustering and exhibits classification ability. ${ }^{(5)}$ In a linear discriminant plot, each point represents the response pattern of a single protease analyte in the PLL-Dnc array. Although slight overlapping of Pap, Pro, and Ela clusters was observed in the plot, there are wellseparated clusters for the other proteases. In order to examine the reliability of the PLL-Dnc array for pattern-recognition-based protease identification, a leave-one-out cross-validation test was performed. For this test, one fluorescence response pattern was removed from the dataset and treated as test data, which was then assigned to the cluster with the closest Mahalanobis distance calculated using the remaining data. This procedure was repeated for all the data. Of the 48 analytes, only two Ela analytes were incorrectly assigned (one to Pap and the other to Pro), providing an accuracy of $98 \%$. This indicates that the PLL-Dnc system has high reliability even for the identification of proteases. 


\subsection{Pattern-recognition-based discrimination of mixed analytes of a protease and its inhibitor protein}

After the successful identification of the proteases, we subsequently investigated whether the PLL-Dnc system could be used to discriminate a composition of mixtures of TryB and its inhibitor protein ATT (Table 1). Because trypsin, which regulates the pancreatic exocrine function, is associated with various diseases, ${ }^{(19)}$ the detection of trypsin and its complex with inhibitor proteins is of diagnostic and clinical significance. ${ }^{(20)}$ Trypsin detection is typically based on the catalytic degradation of the substrate peptides, ${ }^{(21-23)}$ but accurate determination of the concentration is difficult if its activity is altered by the presence of inhibitor proteins. Therefore, we attempted the discrimination of eight different TryB/ATT mixtures, including those with concentration ratios at which the protease activity was completely inhibited by the formation of a 1:1 stoichiometric complex between TryB and ATT [20 $\mu \mathrm{g} / \mathrm{mL}$ TryB $(\sim 0.85 \mu \mathrm{M})$ and $50 \mu \mathrm{g} / \mathrm{mL}$ ATT $(\sim 1.14 \mu \mathrm{M})],{ }^{(11)}$ using the PLL-Dnc array [Fig. 3(a)]. At $\mathrm{pH}=5.5$ and 7.0, only slight changes were observed with the addition of TryB compared with the fluorescence in the absence of proteins, while at $\mathrm{pH}=8.5$, the addition of $10 \mu \mathrm{g} / \mathrm{mL}$ TryB reduced the fluorescence intensity $(0 / 0$ to $0 / 10)$, with the further addition of TryB producing almost no change $(0 / 10$ to $0 / 30)$. The addition of $50 \mu \mathrm{g} / \mathrm{mL}$ ATT markedly increased the fluorescence intensity of PLL-Dnc at all $\mathrm{pH}$ values, which may be mainly due to the electrostatic interaction between the anionic ATT ( $\mathrm{p} I=5.4$; Table 1 ) and cationic PLL-Dnc. Interestingly, when TryB was added to a solution containing ATT, the PLL-Dnc signals decreased regardless of the $\mathrm{pH}$ value.

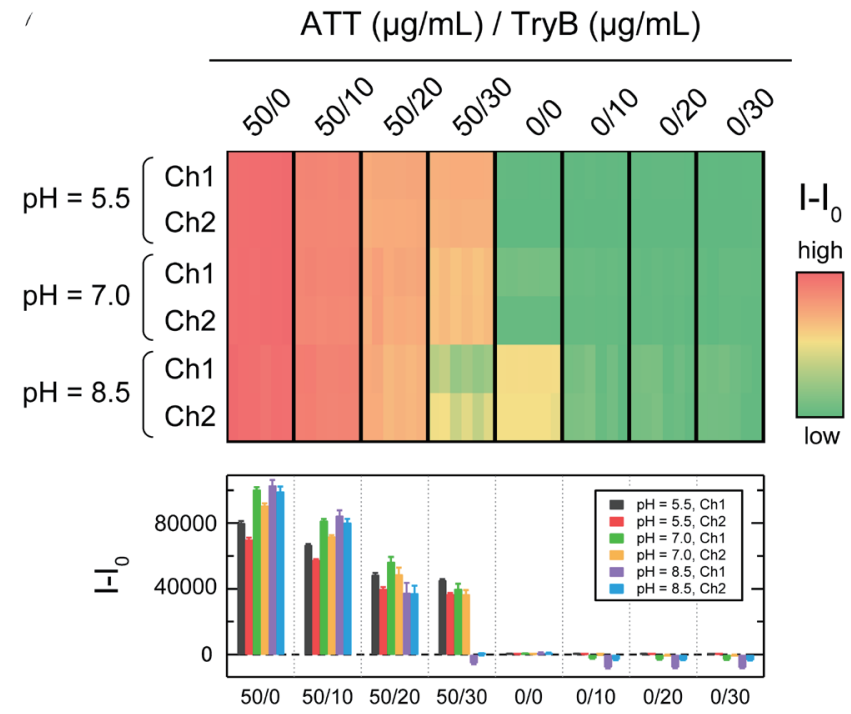

(a)

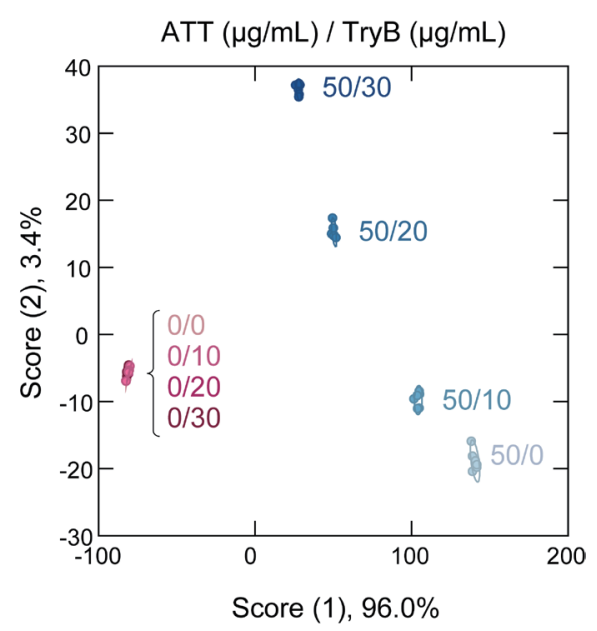

(b)

Fig. 3. (Color online) Discrimination of mixtures of ATT and TryB by an array of PLL-Dnc. (a) Heat map and bar graph of the fluorescence response patterns of various compositions of ATT and TryB. Six replicates are shown for each analyte. (b) Discriminant score plot for the mixtures. Ellipses represent confidence intervals $( \pm 1$ SD) for the individual analytes. 
In the LDA plot of the first two discriminant scores [Fig. 3(b)], the cluster position in the absence of ATT changed little as the concentration of TryB increased ( $0 / 0$ to $0 / 30)$, while the ATT cluster shifted significantly in the negative direction of Score (1) and in the positive direction of Score (2) with an increase in TryB concentration (50/0 to 50/30). This result may be due to a decrease in the exposed surface area as a result of the formation of a complex of ATT and TryB. Despite the overlap of TryB clusters in the plot, the leave-one-out cross-validation test afforded a relatively high value of $90 \%$, suggesting that our PLL-Dnc array is capable of extracting information on the events of the ATT/TryB complex formation as response patterns, although it is currently unable to completely discriminate these eight analytes. Thus, our onecomponent array can be used to discriminate the composition of mixed analytes of a protease and its inhibitor protein. We previously reported the discrimination of ATT/TryB compositions using an array of four fluorophore-modified ssDNAs with different sequences. ${ }^{(11)}$ In this study, we have succeeded in discriminating them using only a single material by utilizing $\mathrm{pH}$-dependent affinities. In addition to the use of different $\mathrm{pH}$ values, the addition of salts to attenuate electrostatic interactions may improve the detection accuracy of the PLL-Dnc system for protease sensing, as we have previously demonstrated. ${ }^{(13)}$ In the case of complex matrices such as serum and urine, PLL-Dnc also interacts nonspecifically with the nontarget proteins contained therein. Although it is still possible to extract target protein information and to accurately identify them using pattern-recognition-based sensing techniques, ${ }^{(24)}$ the introduction of specific recognition units into probes ${ }^{(13,25)}$ or the removal of contaminants by purification will improve the identification accuracy.

\section{Conclusions}

We applied a pattern-recognition-based sensor using PLL-Dnc to the discrimination of proteases. Although our array has high accessibility and consists of only a single material that can be easily prepared, it allowed the identification of various kinds of proteases and the detection of protease/inhibitor protein complexes. Considering that this system can be easily extended through the selection of more diverse solution conditions, e.g., different ionic strengths, ${ }^{(13)}$ our method can potentially be used to screen for the formation of protein-protein complexes, including proteases, as well as to detect disease-associated proteases.

\section{Acknowledgments}

We would like to thank S. Ishihara (Health and Medical Research Institute, National Institute of Advanced Industrial Science and Technology) for technical assistance with the experiments. This work was supported by JSPS KAKENHI grant JP20H02774. 


\section{References}

1 M. Verdoes and S. H. Verhelst: Biochim. Biophys. Acta 1864 (2016) 130. https://doi.org/10.1016/ j.bbapap.2015.04.029

2 A. Dhillon, K. Sharma, V. Rajulapati, and A. Goyal: Current Developments in Biotechnology and Bioengineering: Production, Isolation and Purification of Industrial Products (Elsevier: Amsterdam, 2017) pp. 149-165.

3 R. de la Rica and M. M. Stevens: Nat. Nanotechnol. 7 (2012) 821. https://doi.org/10.1038/nnano.2012.186

4 A. D. Warren, G. A. Kwong, D. K. Wood, K. Y. Lin, and S. N. Bhatia: Proc. Natl. Acad. Sci. U.S.A. 111 (2014) 3671. https://doi.org/10.1073/pnas.1314651111

5 Z. Li, J. R. Askim, and K. S. Suslick: Chem. Rev. 119 (2019) 231. https://doi.org/10.1021/acs.chemrev.8b00226

6 M. De, S. Rana, H. Akpinar, O. R. Miranda, R. R. Arvizo, U. H. F. Bunz, and V. M. Rotello: Nat. Chem. 1 (2009) 461. https://doi.org/10.1021/acs.chemrev.8b00226

7 X. Ran, F. Pu, J. Ren, and X. Qu: Chem. Commun. 51 (2015) 2675. https://doi.org/10.1039/C4CC08863H

8 Q. Xu, Y. Zhang, B. Tang, and C. Y. Zhang: Anal. Chem. 88 (2016) 2051. https://doi.org/10.1021/acs. analchem.5b03109

9 S. Xu, Z. Su, Z. Zhang, Y. Nie, J. Wang, G. Ge, and X. Luo: J. Mater. Chem. B 5 (2017) 8748. https://doi. org/10.1039/C7TB02129A

10 S. Xu, Y. Wu, X. Sun, Z. Wang, and X. Luo: J. Mater. Chem. B 5 (2017) 4207. https://doi.org/10.1039/ C7TB00367F

11 S. Tomita, H. Sugai, M. Mimura, S. Ishihara, K. Shiraki, and R. Kurita: ACS Appl. Mater. Interfaces 11 (2019) 47428. https://doi.org/10.1021/acsami.9b17829

12 M. Okada, H. Sugai, S. Tomita, and R. Kurita: Sensors 20 (2020) 5110. https://doi.org/10.3390/s20185110

13 H. Sugai, S. Tomita, S. Ishihara, and R. Kurita: Sens. Mater. 31 (2019) 1. https://doi.org/10.18494/ SAM.2019.2032

14 H. Sugai, S. Tomita, S. Ishihara, and R. Kurita: ACS Sens. 4 (2019) 827. https://doi.org/10.1021/ acssensors.9b00247

15 O. Hayashida, N. Ogawa, and M. Uchiyama: J. Am. Chem. Soc. 129 (2007) 13698. https://doi.org/10.1021/ ja074906h

16 Y. D. Zhuang, P. Y. Chiang, C. W. Wang, and K. T. Tan: Angew. Chem. Int. Ed. 52 (2013) 8124. https://doi. org/10.1002/ange.201302884

17 A. M. Mallet, Y. Liu, and M. Bonizzoni: Chem. Commun. 50 (2014) 5003. https://doi.org/10.1039/ C4CC01392A

18 Y. Liu and M. Bonizzoni: J. Am. Chem. Soc. 136 (2014) 14223. https://doi.org/10.1021/ja507905r

19 S. Nadalin, P. Girotti, and A. Konigsrainer: Curr. Opin. Organ. Transplant. 18 (2013) 89. http://doi.org/10.1097/ MOT.0b013e32835c6f0f

20 M. Hirota, M. Ohmuraya, and H. Baba: J. Gastroenterol. 41 (2006) 832. https://doi.org/10.1007/s00535-006$\underline{1874-2}$

21 X. Gu, G. Yang, G. Zhang, D. Zhang, and D. Zhu: ACS Appl. Mater. Interfaces 3 (2011) 1175. https://doi. org/10.1021/am2000104

22 Y. Lin, R. Chapman, and M. M. Stevens: Anal. Chem. 86 (2014) 6410. https://doi.org/10.1021/ac500777r

23 D. Zhao, C. Chen, J. Zhao, J. Sun, and X. Yang: Sens. Actuators, B 247 (2017) 392. https://doi.org/10.1016/ j.snb.2017.03.031

24 M. De, S. Rana, H. Akpinar, O. R. Miranda, R. R. Arvizo, U. H. F. Bunz, and V. M. Rotello: Nat. Chem. 1 (2009) 461. https://doi.org/10.1038/nchem.334

25 L. Motiei, Z. Pode, A. Koganitsky, and D. Margulies: Angew. Chem. Int. Ed. 53 (2014) 9289. https://doi. org/10.1002/anie.201402501 